\title{
¿Pueden los indicadores existentes medir el impacto de la crisis económica en los servicios sociales básicos? Un análisis de los indicadores en la provincia de Barcelona (2007-2013)
}

\author{
Núria Fustier García \\ UFR Treball Social, Universitat de Barcelona. \\ nuria.fustier@ub.edu
}

Artikulu hau kokatzen da oinarrizko gizartezerbitzuetako ebaluazioaren arloan, betiere politika sozial izaerako gizarte-zerbitzuen kudeaketarako tresnatzat hartuz eta gogoetarako baliagarri izateko xedez. Erlazionatzen dira gizarte-zerbitzuetako sistema eta 2007-2013 urteen arteko testuinguru sozioekonomikoko adierazleak, modu horretan bi tipologia horien emaitzen arteko erlazioa aztertu eta gizarte-zerbitzuei egokitutako funtzioen betekizuna ebaluatzeko. Halaber, gogoeta burutzen da oinarrizko gizarte-zerbitzuen ekimenen eragina neurtzeko ebaluazioaren gaitasunaz, erabakitzeko garairako informazio garrantzitsua lortzeko gurutzatutako analisiak gehitzearen garrantziaz eta dagokien funtzio eta rolaren arabera erabilitako adierazle horiek oinarrizko gizarte-zerbitzuen eraginkortasuna ebaluatzeko ezaugarriak betetzearen ahalmenaz.

\section{GAKO-HITZAK:}

Gizarte-zerbitzuak, adierazleak, ebaluazioa, politika soziala, krisialdi ekonomikoa.
Este artículo se enmarca en el ámbito de la evaluación de los servicios sociales básicos, como herramienta de gestión y como base para la reflexión sobre los objetivos de los servicios sociales como política social. Se ponen en relación indicadores del sistema de servicios sociales (Círculos de Comparación Intermunicipal de Barcelona) con indicadores del contexto socioeconómico entre 2007 y 2013 para analizar las interrelaciones entre los resultados de ambas tipologías y su adecuación para evaluar el cumplimiento de las funciones asignadas a los servicios sociales. También se reflexiona sobre la capacidad de los indicadores para medir el impacto de la acción de los servicios sociales básicos, sobre la necesidad de incorporar el análisis cruzado, para así obtener información relevante para la toma de decisiones y sobre si los indicadores existentes tienen las características para evaluar la eficacia de los servicios sociales básicos, de acuerdo con las funciones y el rol que se les asigna.

\section{Palabras Clave:}

Servicios sociales, indicadores, evaluación, política social, crisis económica. 


\section{Introducción}

En junio del año 2000 se celebró en Madrid la Conferencia Europea de Servicios Sociales, organizada por la European Social Network, dedicada a la calidad de los servicios sociales, y allí conocimos experiencias de benchmarking en el ámbito de los servicios sociales. Ese ejemplo motivó a la Diputación de Barcelona y a diversos directivos locales de servicios sociales, y en 2001 empezó la experiencia de los Círculos de Comparación Intermunicipal (Cercles, en adelante) con 25 municipios, y entre ellos, el municipio en el que yo trabajaba. Fue un proceso muy interesante porque la primera tarea fue diseñar unos indicadores útiles y posibles con los sistemas de información que disponíamos. Mi relación con los sistemas de indicadores de servicios sociales continúa desde entonces, desde distintas experiencias y actividades profesionales.

Desde los inicios los Cercles han tenido un principio inspirador, "Lo que no se puede medir, no se puede evaluar y lo que no se puede evaluar, no se puede mejorar", y durante todo este tiempo los indicadores nos han mostrado la evolución que los servicios sociales vivían, aunque centrándose en una comparación interna: resultados de los mismos indicadores a lo largo del tiempo.

En plena crisis, cuando los servicios sociales se vieron abocados a una mayor presión para dar respuesta a las necesidades crecientes derivadas, sobre todo, de la pérdida de los puestos de trabajo y sus efectos sobre la vivienda y la cobertura de otras necesidades básicas, me planteé si estos indicadores eran, de alguna manera, sensibles a los cambios en el sistema económico y qué análisis podríamos hacer si relacionábamos indicadores económicos con nuestro sistema de indicadores.

Así pues, me planteé un estudio (Fustier, 2015) que tenía por objetivo general "identificar y seleccionar los indicadores que pueden medir el impacto de la crisis en los servicios sociales municipales". Para ello:

- Analicé diversos sistemas de indicadores de servicios sociales: los indicadores de los Cercles, los indicadores de los Planes de Actuación Local en materia de Servicios Sociales (PALMSS) de la Generalitat de Catalunya y los indicadores del Informe DEC (Asociación Española de Directoras y Gerentes de Servicios Sociales).

- Escogí un período que abarcara distintos momentos del desarrollo económico: 2007-2013.

- Seleccioné un territorio que presentara una cierta homogeneidad en cuanto al desarrollo de los servicios sociales: la provincia de Barcelona.

Finalmente, opté por escoger los indicadores de los Cercles porque eran los únicos que tenían una batería estable de indicadores para todo el periodo, lo que cumplía el objetivo de comparar periodos económicos distintos y el comportamiento de los indicadores de los servicios sociales en estas situaciones. El estudio analiza la interrelación entre los resultados de los indicadores de servicios sociales y los de indicadores macroeconómicos para identificar si las variaciones que se muestran responden a una política social orientada a ofrecer una mayor protección en momentos de mayor vulnerabilidad y, en su caso, quién es el último responsable de dicha protección.

Este artículo es un resumen del estudio y se estructura en cuatro apartados y las conclusiones: el primer apartado está dedicado al marco conceptual, tanto de los servicios sociales de Catalunya como del propio concepto de crisis económica; el segundo y tercero se centran en la presentación de los resultados de los indicadores de servicios sociales y macroeconómicos; en el cuarto se analiza la interrelación entre las diversas magnitudes de indicadores; $y$, finalmente, las conclusiones presentan el resultado del análisis cruzado de indicadores sociales y económicos.

\section{Marco conceptual}

\subsection{Los servicios sociales en Cataluña}

La actual regulación de los servicios sociales mediante la Llei 12/2007, d'11 d'octubre, de Serveis Socials, se enmarca en el nuevo Estatuto de Autonomía (Ley Orgánica 6/2006) que reconoce, en su artículo 24, el derecho a los servicios sociales.

La ley define el objeto y la finalidad de los servicios sociales en un sentido muy amplio ${ }^{1}$; el artículo 3 lo recoge de la siguiente manera:

1. Los servicios sociales tienen como finalidad asegurar el derecho de las personas a vivir dignamente durante todas las etapas de la vida mediante la cobertura de sus necesidades personales básicas y de las necesidades sociales, en el marco de la justicia social y del bienestar de las personas.

2. Son necesidades sociales, a los efectos de lo que establece el apartado 1 , las que repercuten en la autonomía personal y el apoyo a la dependencia, en una mejor calidad de vida personal, familiar y de grupo, en las relaciones interpersonales y sociales y en el bienestar de la colectividad. Las necesidades personales básicas son las propias de la subsistencia y la calidad de vida de cada persona. (Llei 12/2007, d'11 d'octubre, de Serveis Socials, art. 3.1 y 3.2.)

${ }^{1}$ Es habitual que tanto refiriéndose al Estado de Bienestar como a los servicios sociales se den dos acepciones: una amplia y una restringida. La primera, en el caso de los servicios sociales, los identifica con el conjunto de servicios de protección social (sanidad, educación, pensiones...) y en sentido restringido se refiere a uno de los sistemas de protección social, el de la asistencia social (Gómez i Serra, 2000: 48). 
Destacamos las principales características de esta ley:

- La inclusión del principio de universalidad, diferenciado de la gratuidad, especificando que su sentido se refiere a que toda persona que necesite un servicio debe tener acceso.

- La regulación de derechos subjetivos y deberes de las personas en relación con los servicios sociales, a los que se dedica buena parte del Título I. Se destaca por ser la primera vez que se incluyen en una norma de máximo rango legal, ya que anteriormente quedaban recogidos en normas de rango inferior.

- Ámbito de actuación: la ley introduce un listado de situaciones de atención prioritaria (artículo 7) que limitan el alcance de la universalidad.

- La estructura del sistema en dos niveles: servicios sociales básicos y servicios sociales especializados.

- La asignación de competencias de las administraciones: el Gobierno y la Administración de la Generalitat se reservan las competencias vinculadas al desarrollo normativo, planificación general, fijación de criterios y estándares para su desarrollo, así como la inspección, el control y la autorización; asimismo, se reserva la gestión de los servicios sociales especializados. Por su parte, otorga la competencia sobre los servicios sociales básicos a los municipios y a los consejos comarcales.

- Planificación: se prevé la elaboración cuatrienal de un plan estratégico, basado en una evaluación de la situación de los servicios sociales y vinculado a un plan de calidad. Hasta el momento solo se ha elaborado y aprobado el I Plan Estratégico de Servicios Sociales de Cataluña, para el período 2010-2013.

La atención básica se conceptualiza como un “dispositivo potente de información, diagnóstico, orientación, apoyo, intervención y asesoramiento individual y comunitario", de "carácter público y local" y que se configura como "la puerta de entrada habitual al sistema" y “eje vertebrador de toda la red de servicios” (Ley 12/2007: preámbulo).

Los servicios sociales básicos se regulan en dos artículos (arts. 16 y 17). El primero incluye la definición básica, cuyos componentes son una organización de base territorial, equipos multidisciplinarios, estructura directiva y de apoyo técnico y administrativo.

En este nivel se incluyen, a falta de desarrollar la cartera, “los equipos básicos, los servicios de ayuda a domicilio y de teleasistencia y los servicios de intervención socioeducativa no residencial para la infancia y adolescencia” (art.16.2) que actúan, con "carácter polivalente, comunitario y preventivo para fomentar la autonomía de las personas" (art. 16.3), sobre la base territorial de los municipios de referencia. No obstante, "la regulación no se acaba en la definición y en una primera enumeración de las principales prestaciones incluidas, sino que, al contrario de la regulación anterior, la nueva norma incluye un extenso listado de las funciones de los servicios sociales básicos" (Matulič y Fustier, 2008).

\subsection{Crisis económica}

Una crisis económica es "una situación en la que se producen cambios negativos importantes en las principales variables económicas, y especialmente en el PIB y en el empleo" (Uxó González, s/f). Uxó González apunta que "la expresión crisis económica debe reservarse para aquellas situaciones en las que las dificultades económicas van más allá de un simple empeoramiento transitorio" y distingue tres grados de severidad -desaceleración, recesión y depresión- que se distinguen por la profundidad y sostenimiento de la reducción de la tasa de crecimiento hasta tasas negativas. Una crisis económica "afecta de manera generalizada al conjunto de la economía (no solo a algunos sectores)" y a la caída del PIB le acompañan importantes descensos en las tasas de empleo y caída del consumo que, a su vez, afectará a la tasa de inflación, que puede llegar a ser negativa, convirtiéndose en deflación.

En relación con las variables que permiten medir la actividad económica y, por tanto, identificar situaciones de crisis o bonanza, diversos autores consultados señalaban distintas variables. Así, Uxó González identifica cuatro variables: PIB, empleo, inflación y consumo. Rosende y Bengolea (1984) recogen tres: el sistema de precios (inflación), la confianza para la inversión y el valor del dinero (interés). Krugman (2013) recoge la demanda y el crecimiento demográfico.

\section{Los indicadores de los Círculos de Comparación Intermunicipal de Servicios Sociales}

\subsection{Una breve introducción a los Círculos}

Los Círculos de Comparación Intermunicipal de Servicios Sociales (CCI) son grupos estables de responsables técnicos de servicios sociales municipales que, con la colaboración y liderazgo de la Diputación de Barcelona, pretenden avanzar hacia una gestión de calidad de los servicios públicos municipales.

Los Círculos de Comparación de Servicios Sociales se iniciaron en el año 2001 con la participación de 25 municipios voluntarios que elaboraron una primera batería de 24 indicadores. En 2007, con el número de municipios participantes estabilizado en $53-55^{2}$, que

${ }^{2}$ Los municipios participantes son mayoritariamente de la provincia de Barcelona, aunque en la actualidad hay cuatro municipios de otras demarcaciones, donde no existe la experiencia y que, por sus características, tampoco tendrían municipios cercanos similares para 
representan en torno al 60\%-70\% de la población de la provincia, se revisa y se elabora una guía que contiene 66 indicadores estructurados en cuatro dimensiones (Diputació de Barcelona, 2013, 2014; Fustier, 2006, 2008):

- Estratégica o encargo político: relacionada con la consecución de los objetivos finales del servicio y de su calidad. Formada por 25 indicadores.

- Persona usuaria o cliente: relacionada con el uso y la satisfacción que las personas usuarias tienen respecto de los servicios. Formada por 11 indicadores.

- Valores organizativos/recursos humanos: relacionada con el modelo organizativo, de gestión y los recursos humanos. Formada por 13 indicadores.

- Económica: relacionada con el uso de recursos necesarios para llevar a cabo el servicio, sus costes asociados y las fuentes de financiación. Formada por 20 indicadores.

Antes de pasar al análisis, debemos señalar que un aspecto clave de los indicadores reside en los sistemas de información y estos mejoran muy lentamente. Este hecho puede conllevar que, en algún caso, las variaciones de resultados entre un ejercicio y otro no respondan a cambios reales, sino al propio sistema de información.

Los indicadores, clasificados por dimensiones, se centran en las prestaciones definidas en la Cartera de Servicios Sociales (Departament d’Acció Social i Ciutadania, 2010) para el nivel de los servicios sociales básicos, y para procurar obtener resultados comparables se han fijado las condiciones siguientes:

- Homogeneidad de las prestaciones. Existencia entre los participantes de uno, o a lo sumo dos, modelos teóricos o de gestión de dicha prestación claramente identificados.

- Perdurabilidad de las prestaciones. Deben ser prestaciones consolidadas, vinculadas a las funciones básicas y a las prestaciones esenciales, que formen parte del eje vertebrador de la cartera.

- Existencia de información clave común. Si coexisten distintos modelos de gestión, se requiere la existencia de información relevante (indicadores) y cuyos resultados puedan ser comparables entre distintas modalidades de prestación. De hecho, esta característica dotaría al indicador de un valor añadido en la medida en que contribuiría a la toma de decisiones sobre la modalidad más efectiva y eficaz mediante la comparación.
Estas condiciones limitan el desarrollo de indicadores para todas las prestaciones, puesto que una parte de ellas presentan múltiples formas de gestión que difícilmente son comparables entre ellas ${ }^{3}$. Así pues, partiendo del análisis de la cartera, y respetando las condiciones señaladas, se han definido indicadores para las siguientes prestaciones y servicios:

- Servicios sociales básicos. Incluye los indicadores para el conjunto de los servicios, indicadores globales que tienen que ver con la estructura o que no pueden ser individualizados por cada prestación.

- Servicio básico de atención social. Este servicio se corresponde con la prestación de los equipos profesionales de atención social.

- Prestaciones económicas de urgencia social.

- Servicios sociales de atención domiciliaria. Estos servicios se concretan en dos prestaciones distintas: servicio de ayuda a domicilio y servicio de teleasistencia; también existen indicadores del conjunto de ambos servicios.

- Centros abiertos. Se trata de uno de los servicios más estables de la categoría de "Servicios de intervención socioeducativa no residencial para niños y adolescentes" prevista en la cartera.

\subsection{Principales resultados y evolución en el periodo 2007-2013}

El estudio seleccionó 29 indicadores del total de 66 de la batería. La selección se hizo sobre la base de que las variables y el resultado tuvieran relación directa con los elementos vinculados con la crisis; así pues, se seleccionaron indicadores económicos, indicadores de estructura organizativa, indicadores de cobertura e indicadores de intensidad.

El análisis se realiza a partir de la media global y de las medias por grupos de población ${ }^{4}$. Los indicadores seleccionados clasificados en función de las dimensiones son los que recoge la Tabla 1.

En el proceso del análisis cruzado de los resultados de los indicadores de los servicios sociales y las variables socioeconómicas se realizó una segunda selección en función de la posibilidad de interrelacionar los dos tipos resultados. Esta segunda selección redujo el número de indicadores a 13 y se distribuyeron tal como se refleja en la Tabla 2 , siendo estos los indicadores que se analizarán en este apartado.
${ }^{3}$ La propia cartera de servicios sociales permite una variada forma de gestión de algunas prestaciones.

4 Existen cuatro círculos según el tamaño del municipio: mayores de 70.000 habitantes, mayores de 40.000 habitantes (de 40.000 a 69.999), mayores de 20.000 habitantes (de 20.000 a 39.999) y mayores de 10.000 habitantes (de 10.000 a 19.999). 
Tabla 1. Distribución de los indicadores estudiados según las dimensiones definidas por los Círculos de Comparación

\begin{tabular}{|c|c|c|c|c|}
\hline & $\begin{array}{l}\text { Dimensión } \\
\text { estratégica }\end{array}$ & $\begin{array}{l}\text { Dimensión de } \\
\text { usuario }\end{array}$ & $\begin{array}{l}\text { Dimensión de recursos } \\
\text { humanos- organizativa }\end{array}$ & $\begin{array}{l}\text { Dimensión } \\
\text { económica }\end{array}$ \\
\hline Servicios sociales básicos & 2 & & & 2 \\
\hline Servicios básicos de atención social & 5 & & & 2 \\
\hline Ayudas de urgencia social & & & & 2 \\
\hline Servicios sociales de atención domiciliaria & 5 & 1 & & 2 \\
\hline Centros abiertos & 1 & 1 & 1 & 2 \\
\hline
\end{tabular}

Fuente: Elaboración propia.

Tabla 2. Distribución de los indicadores sujetos a comparación con variables socioeconómicas por dimensiones

\begin{tabular}{|c|c|c|c|c|}
\hline & $\begin{array}{l}\text { Dimensión } \\
\text { estratégica }\end{array}$ & $\begin{array}{c}\text { Dimensión de } \\
\text { usuario }\end{array}$ & $\begin{array}{l}\text { Dimensión de recursos } \\
\text { humanos- organizativa }\end{array}$ & $\begin{array}{l}\text { Dimensión } \\
\text { económica }\end{array}$ \\
\hline Servicios sociales básicos & 2 & & & 2 \\
\hline Servicios básicos de atención social & 3 & & & \\
\hline Ayudas de urgencia social & & & & 1 \\
\hline Servicios sociales de atención domiciliaria & 1 & 1 & & 2 \\
\hline Centros abiertos & 1 & & & 1 \\
\hline
\end{tabular}

Fuente: Elaboración propia.

\subsubsection{Servicios sociales básicos (SSB)}

Se han seleccionado cuatro indicadores para los servicios sociales básicos (SSB): peso de los SBB en el presupuesto municipal, gasto corriente en SSB por habitante, grado de autofinanciación mediante tasas y precios públicos y grado de financiación externa finalista.

El peso de los servicios sociales básicos en el presupuesto municipal muestra "la importancia relativa otorgada a los servicios sociales respecto del presupuesto total del ayuntamiento" (Diputació de Barcelona, 2014). Los resultados muestran una tendencia incrementalista en el periodo estudiado, que se concreta en un aumento del $71 \%$ entre 2007 y 2013. Por tamaños, los municipios con más habitantes dedican más presupuesto, seguidos de los municipios mayores de 20.000 habitantes. El grupo de los municipios más pequeños es el que destina menor presupuesto, aunque debe señalarse que no están obligados legalmente a prestarlos.

En consonancia con lo anterior, el gasto corriente por habitante, que excluye inversiones y gastos financieros, muestra también una tendencia al incremento, aunque presenta un cierto estancamiento en el periodo 2010-2013; en el conjunto del periodo se produce un incremento del $37 \%$, siendo los municipios más pequeños los que mayor crecimiento relativo presentan.

Los otros dos indicadores estudiados analizan la estructura de financiación de los servicios sociales básicos: la financiación por tasas y precios públicos y la financiación por aportación de otras Administraciones. El primero, tasas y precios públicos, aunque tiene un peso muy bajo en los presupuestos municipales, no llegando la media al $3 \%$, muestra el impacto del copago de los servicios y mantiene una tendencia incrementalista durante todo el periodo, aunque irregular: en el primer periodo (2007-2010) aumenta un $83 \%$ de media, mientras que en el segundo (2010-2013) el aumento es solo de un $8 \%$.

El indicador de financiación externa refleja el peso de las aportaciones de otras Administraciones para la financiación finalista de los servicios sociales básicos. Este indicador mide, fundamentalmente, la aportación de la Generalitat y el cumplimiento de lo establecido en la ley al respecto. En cuanto a los resultados, se observa un incremento en el periodo 2007-2010 y una caída en el siguiente ejercicio, con una recuperación leve en los años siguientes, quedando ligeramente por encima del $45 \%$. La interpretación conjunta de los indicadores de financiación muestra que más de la mitad del presupuesto de servicios sociales proviene del propio municipio, cuando la ley establece que debería cubrir el $33 \%$.

\subsubsection{Servicio básico de atención social}

La cobertura del servicio básico de atención social (SBAS) mide la capacidad del servicio para atender a la población; en el periodo de estudio se observa un incremento neto que ronda el $60 \%$, aunque presenta un cierto estancamiento desde el año 2011. Los resultados muestran un incremento homogéneo en todos los municipios, aunque inversamente proporcional al tamaño, es decir, a menor tamaño, mayor cobertura y mayor incremento. La existencia de una mayor variedad y accesibilidad de servicios a las personas en los municipios mayores es una de las explicaciones que se consideran para esta tendencia.

La renta mínima de inserción es una prestación clave, especialmente si se estudia el impacto de la crisis económica en la vida de las personas, puesto que representa la última red asistencial, tras el 
agotamiento de las prestaciones de desempleo o la imposibilidad de acceder a las pensiones. La cobertura muestra una curva ascendente hasta el año 2010, llegando en este ejercicio a doblar la cobertura de 2007 y, a partir de este momento, sufre una caída del $7 \%$ que inicia la tendencia de retroceso en la cobertura y alcanza, en 2013 un descenso del $14 \%$ respecto a 2010.

El último indicador estudiado relaciona las personas atendidas con el número de profesionales de los SBAS. Entre 2007 y 2008 se produce un leve descenso ( $5 \%$ ) que se corresponde con el incremento de plantillas previsto en la Ley de 2007 , pero a partir de este momento la tendencia al alza es continuada y el mantenimiento de las plantillas permite absorber la mayor demanda, aunque con niveles elevados de atención profesional, puesto que el incremento en el conjunto del periodo es del $12 \%$.

\subsubsection{Prestación económica de urgencia social}

La prestación económica de urgencia social está contemplada en la cartera de servicios y es objeto de regulación en una norma específica 5 . Los Círculos incorporaron indicadores sobre esta prestación en 2010. Se ha seleccionado el indicador que muestra la distribución del presupuesto destinado a este fin: gasto corriente destinado a ayudas de urgencia social por unidad de convivencia beneficiaria.

Este indicador se define por unidades familiares y esto se debe a que las prestaciones de urgencia se destinan a hacer frente a gastos no individuales (vivienda, suministros, etc.). La tendencia creciente se mantiene en el periodo estudiado, alcanzado un incremento de prácticamente el $30 \%$.

\subsubsection{Servicios sociales de atención domiciliaria}

El primer indicador es el de cobertura del conjunto de los servicios sociales de atención domiciliaria (SSAD) sobre el grupo de edad de 65 años o más. La evolución de los resultados de este indicador muestra un crecimiento global en torno al $50 \%$, aunque con un comportamiento irregular: entre 2007 y 2010 se produce un aumento sostenido que alcanza prácticamente el $60 \%$, para entrar posteriormente, en un periodo más estable con ligeros incrementos y decrementos. La cobertura de estos servicios es superior en los municipios de menor tamaño.

El indicador de accesibilidad mide un aspecto sensible a la situación económica: el grado de exención del copago en los servicios de atención domiciliaria, es decir, el porcentaje de personas que no deben pagar las tasas públicas debido a su situación económica. Este indicador, que se obtiene

\footnotetext{
${ }_{5}^{5}$ Véase el artículo 30 de la Ley 13/2006, de 27 de julio, de prestaciones sociales de carácter económico; Diario Oficial de la Generalitat de Catalunya, número 4.691, 4 de agosto de 2013.
}

desde 2010, muestra una curva con un punto de inflexión: en 2011 se produjeron los mayores niveles de exención del copago y, desde entonces, el grado de cobertura no deja de bajar, llegando en 2013 a niveles inferiores a los de $\mathbf{2 0 1 0}$, con un resultado de poco más de la mitad de la población exenta del copago (un $53,4 \%$ ), mientras que se había alcanzado el $64 \%$ en el momento de mayor población exenta.

Finalmente, se han seleccionado dos indicadores de carácter económico: el gasto corriente por habitante destinado a servicios sociales de atención domiciliaria y el precio medio de hora de servicio de ayuda a domicilio externalizado.

En cuanto a los recursos destinados a los servicios de atención domiciliaria, la tendencia es creciente, duplicándose en el periodo, de los 8,30 euros de 2007 a los 16,30 euros de 2013, aunque el incremento importante se produce entre $2007 \mathrm{y}$ 2010, cuando alcanza el $90 \%$, mientras que a partir de 2010 el crecimiento se estanca y alcanza el $10 \%$ restante, manteniéndose en torno a los 16 euros.

Por último, el precio medio por hora contratada externamente es un indicador relevante porque repercute directamente en los presupuestos municipales y en la calidad del empleo, ya que buena parte del coste del precio por hora se destina al pago de los salarios del personal de atención directa ${ }^{6}$. El precio por hora se ha visto incrementado en el periodo 2007-2011, alcanzando su máximo en 16,5 euros en ese año (lo que supuso un incremento del $20 \%$ ) para después descender ligeramente hasta los 16,3 euros.

\subsubsection{Centros abiertos}

Para los centros abiertos (servicios de intervención socioeducativa no residencial para niños y adolescentes) se han escogido dos indicadores que miden la cobertura de la oferta y el gasto corriente. El primer indicador es la cobertura de la oferta de plazas de centro abierto; para ello se toma como población de referencia la población diana, que, a partir del Plan Estratégico de Servicios Sociales de Catalunya (Maluquer et al., 2010: 258), se identificó con la población de o a 17 años.

El resultado medio de este indicador prácticamente no presenta variaciones, moviéndose entre valores por debajo de las 7 plazas por cada 1.000 habitantes, con excepción del último año estudiado, cuando sufre un incremento significativo, pasando de 6,4 a 7,7 plazas por 1.000 habitantes.

Por su parte, el indicador económico de gasto corriente por habitante, que se referencia a la población diana, muestra el esfuerzo de los

${ }^{6}$ Un estudio de Deloitte sobre el servicio de atención domiciliaria calculaba que el peso de los costes salariales suponía un $77 \%$ del coste total por hora en Cataluña (Deloitte, 2008: 49). 
municipios para prestar estos servicios. Los resultados muestran un proceso de incremento entre 2008 y 2009 , cuando alcanza el resultado más alto $(24,1$ euros por habitante), para después iniciar un fuerte descenso que le lleva a situarse en 2013 en 10,5 euros, lo que significa que el esfuerzo municipal se ha reducido a más de la mitad entre 2009 y 2013

\section{Indicadores macroeconómicos y socioeconómicos}

De esta batería de indicadores se seleccionaron cinco para el análisis cruzado de datos con los indicadores de servicios sociales:

- Evolución del PIB.

- Tasa de paro.

- Gasto medio familiar.

- Tasa de riesgo de pobreza.

- Viviendas según ocupación.

\subsection{Producto interior bruto}

El producto interior bruto (PIB) “mide el resultado final de la actividad de producción de las unidades productoras en el territorio" (Idescat, 2014). La forma más habitual de estudiar esta magnitud es el análisis de la variación que sufre el valor en un periodo de tiempo y se expresa en porcentaje de variación.

La evolución de la economía en el periodo de estudio (2007-2013) partía de un nivel positivo del PIB (+2,9 $y+3,6$ en Catalunya y España, respectivamente) pero rápidamente se inicia un profundo descenso que se extiende, con la excepción de una leve recuperación de 2010 (+0,4 y +0,5 en Catalunya y España, respectivamente) durante todo el periodo en la banda negativa, alcanzando en 2009 el peor resultado $(-4,4$ $\mathrm{y}-3,8$, respectivamente). Aun presentando resultados ligeramente distintos, las curvas de Cataluña y España son prácticamente idénticas.

\subsection{Empleo}

Con relación al mercado de trabajo, existen distintos indicadores que informan sobre la evolución de la economía. Entre los más habituales se encuentran las tasas de ocupación, de actividad y de paro7. La principal fuente de información sobre estos tres indicadores es la Encuesta de Población Activa (EPA)

7 De acuerdo con Idescat (2014) se define población ocupada como el conjunto de personas de 16 años de edad o más y que están trabajando, sea por cuenta ajena o por cuenta propia. Es población desocupada aquella que tiene 16 años de edad o más y que no tiene empleo, aunque está disponible para trabajar y busca trabajo de forma activa. Y es población activa la formada por el conjunto de la población ocupada y desocupada. Por lo tanto, está formada por el conjunto de personas de 16 años de edad o más que está trabajando o en disponibilidad de trabajar. que el Instituto Nacional de Estadística (INE) realiza trimestralmente, aunque sobre la tasa de paro se puede utilizar el indicador de paro registrado que se obtiene del Sistema de Empleo Público Español. La tasa de desempleo en Catalunya muestra un incremento sostenido, multiplicándose por 3,4 en el periodo estudiado (de 6,66\% a $22,55 \%$ ).

\subsection{Presupuestos familiares}

El INE realiza periódicamente encuestas sobre presupuestos familiares que permiten obtener información sobre la disponibilidad de renta y el consumo que realizan los hogares españoles. Uno de los indicadores más interesantes para el propósito del análisis es el gasto medio por vivienda, que mide "el gasto realizado por los hogares en bienes y servicios que se utilizan para satisfacer directamente las necesidades o carencias de los miembros del hogar" (INE, 2007). La evolución de los gastos medios familiares en el periodo 2007-2013 no ha dejado de descender, llegando a reducirse en un $14 \%$.

\subsection{Pobreza relativa}

La pobreza relativa es un concepto que permite una aproximación del fenómeno de la pobreza; se parte del establecimiento del umbral de la pobreza, aquella cuantía de ingresos por debajo de los cuales se considera que existe riesgo de pobreza, que se ha fijado en el $60 \%$ de la renta mediana. El porcentaje de personas que se encuentran por debajo del umbral es la tasa de riesgo de pobreza.

Finalmente, cabe destacar que, dado que el umbral se fija para una sociedad determinada, es habitual que se presenten diferencias entre los umbrales de territorios distintos dentro de un mismo país; así, por ejemplo, el umbral de la pobreza del conjunto de España es inferior al de Catalunya y, por ello, las tasas de riesgo de pobreza pueden sufrir variaciones según se tome uno u otro umbral.

La tasa de riesgo de pobreza muestra el porcentaje de población que se encuentra bajo el umbral de pobreza. La evolución en el periodo muestra una ligera tendencia al alza, no llegando al $10 \%$ de incremento, pero se debe tener en cuenta que cualquier incremento o descenso generalizado de las rentas comporta una modificación del umbral y, cuanto más bajo esté, más personas quedarán fuera (es decir, sin una situación de pobreza relativa).

\subsection{Viviendas familiares según actividad}

Para finalizar este bloque, un indicador que refleja la actividad económica traducida en puestos de trabajo: las viviendas familiares según la actividad de sus miembros. Se obtiene de la Encuesta de Población Activa y muestra, entre los hogares con un mínimo 
de un miembro activo ${ }^{8}$, aquellos que tienen todos los miembros activos ocupados o todos desocupados.

Este indicador señala el impacto del fenómeno del paro en la población en general, pero también su impacto agregado en unidades familiares. La evolución de la crisis ha conllevado un aumento muy importante de las viviendas con todos los miembros activos desocupados 9 , que en número absolutos se ha multiplicado por 5,5 entre 2007 y 2013. Por otra parte, se han incrementado las viviendas familiares sin activos un $18,8 \%$, mientras que han disminuido las viviendas con todos los activos ocupados en un $19,5 \%$.

\section{Comparación entre los resultados de los indicadores del sistema de servicios sociales y los indicadores macroeconómicos}

En este apartado se analizan los resultados de los indicadores seleccionados relativos a los servicios sociales locales en relación con los indicadores macroeconómicos y socioeconómicos, para intentar descubrir qué relaciones se pueden observar en las tendencias de los diversos indicadores con la finalidad de identificar el grado de sensibilidad de los indicadores para explicar el papel de los servicios sociales ante la crisis.

\subsection{Servicios sociales básicos}

Para el conjunto de los servicios sociales básicos, vamos a realizar el análisis de los indicadores de PIB y tasa de paro con relación al esfuerzo económico de los municipios en servicios sociales y el del gasto medio por familia con el de estructura presupuestaria.

Se observa que las representaciones gráficas correspondientes al esfuerzo realizado por los municipios, tanto en términos absolutos (gasto corriente por habitante) como en términos relativos (peso en el presupuesto municipal), divergen de la tendencia mantenida por la variación del PIB; mientras que los primeros muestran una tendencia al aumento, el segundo muestra una tendencia a la baja. Se ha añadido la tasa de paro para ver que muestra una curva muy similar a la de los indicadores de los servicios sociales. Debemos señalar que el nivel de incremento es muy distinto: mientras que la tasa de paro se multiplica por 3,4, los presupuestos se incrementan alrededor de un $40 \%$ (en gasto por habitante). Podríamos deducir que, ante una mayor ralentización de la economía que comporta un mayor índice de desocupación, los servicios sociales han reaccionado incrementando sus recursos para hacer frente a las situaciones de necesidad, pero dado que forman parte también de la misma economía ralentizada, no han crecido al mismo ritmo que la desocupación lo hacía.

Gráfico 1. Interrelación entre el esfuerzo económico municipal en servicios sociales y la evolución del PIB y la tasa de paro en Cataluña durante el periodo 2007-2013

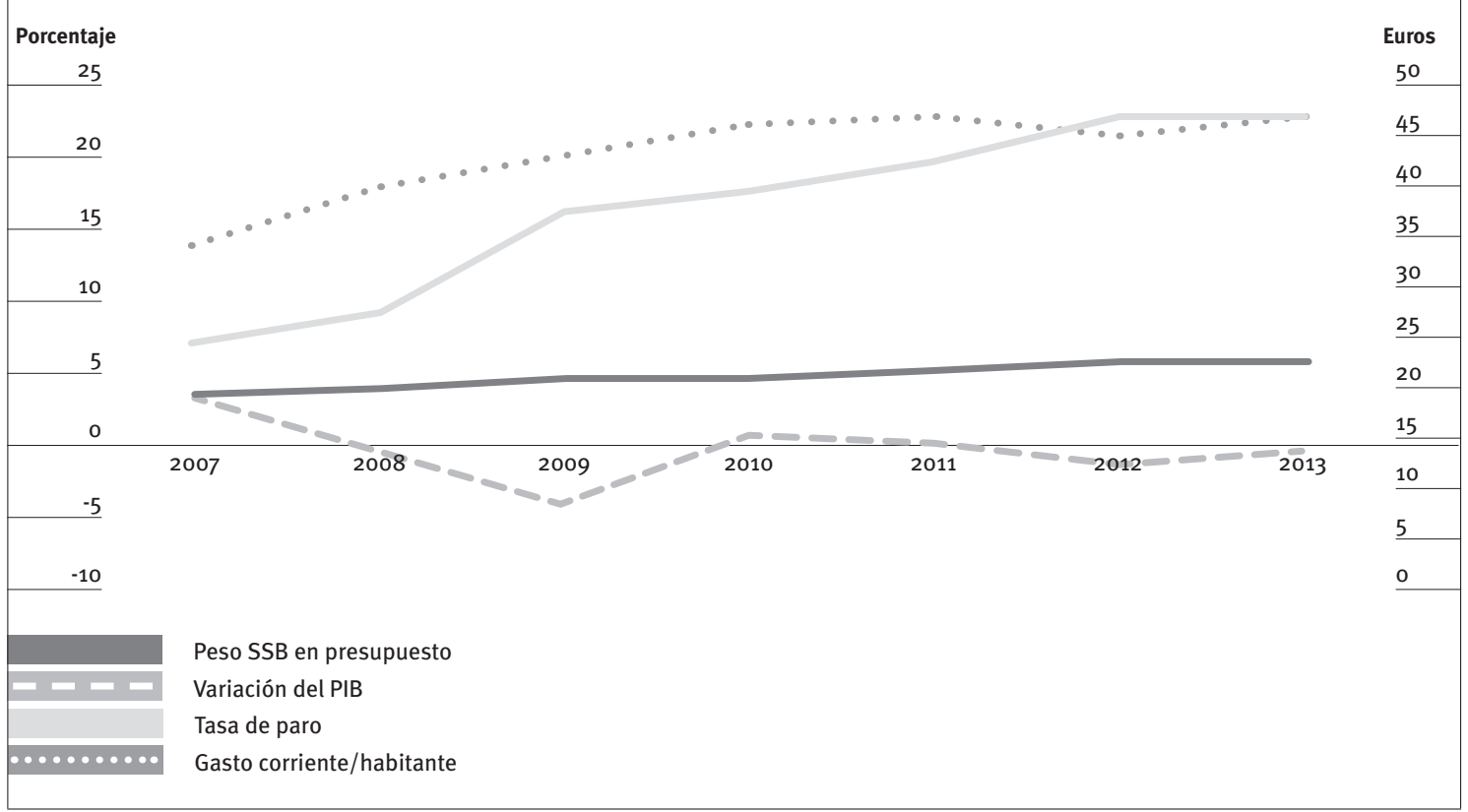

Fuente: Elaboración propia.

${ }^{8}$ Es decir, en edad laboral, a partir de los 16 años, que trabaja 0 está disponible para hacerlo.

9 Se identifica con una línea para permitir una mayor visibilidad y se referencia al eje de la derecha. 


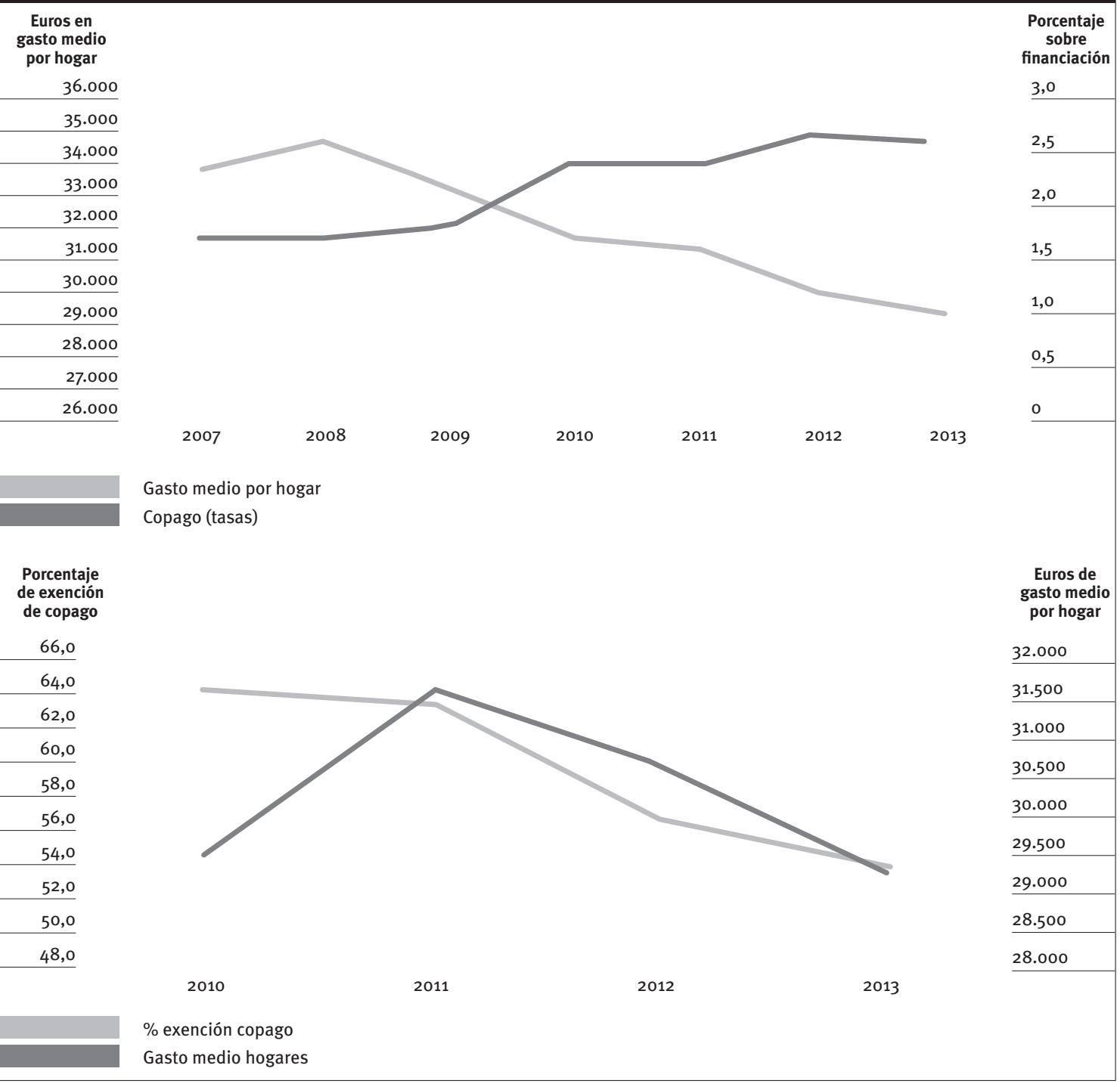

Fuente: Elaboración propia.

Otro análisis interesante es la interrelación entre la estructura de financiación de los servicios sociales y el gasto medio por familia. Sin olvidarnos de la curva que dibuja la tasa de desocupación del gráfico anterior, observamos que el gasto medio de las familias presenta una tendencia descendente, coherente con el aumento de la tasa de paro. Lo destacado es que a menor gasto medio por familia se corresponde una mayor aportación a los servicios sociales mediante el copago: prácticamente se ha doblado la aportación. Podemos observar en el Gráfico 2 que la tendencia del porcentaje de financiación por tasas y precios públicos (copago) en los servicios sociales básicos es opuesta a la línea dibujada por los valores del gasto medio familiar (gráfico superior). También se puede ver, en el gráfico inferior, que el porcentaje de personas que consiguieron una exención al copago ha disminuido a partir de 2011, justo cuando se produce la mayor disminución en el gasto medio de los hogares.

\subsection{Servicios básicos de atención social}

En este apartado se analizará la relación entre la cobertura de los servicios básicos de atención social y de la Renta Mínima de Inserción con la evolución de la tasa de desempleo en primer lugar y, a continuación, se analizará la interrelación entre la misma tasa de desempleo y la carga asistencial de los profesionales del servicio básico de atención social.

En el Gráfico 3 se puede ver la relación entre la evolución de la tasa de desempleo con la cobertura del Servicio Básico de Atención Social (SBAS) y la de Renta Mínima de Inserción (RMI). Se observa que en el periodo 2007-2010, con el mayor incremento de la tasa de desempleo, se produjo también un mayor incremento en la respuesta del sistema de servicios sociales (ambas prestaciones), pero a continuación, mientras que la tasa de desempleo sigue aumentando, la cobertura de RMI sufre un claro 


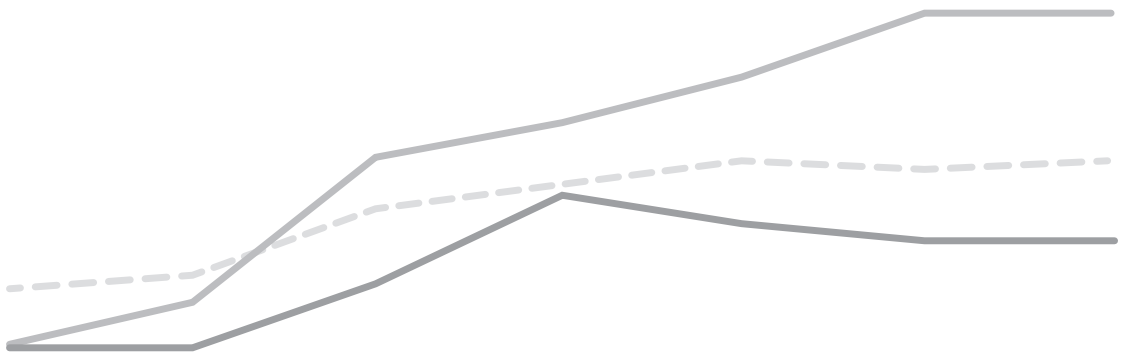

Fuente: Elaboración propia.

parón en 2011 y un retroceso en los años posteriores. Por su parte, la cobertura de SBAS se mantiene con una tendencia de aumento sostenido, aunque más de los servicios básicos de atención social en la ley pero, como se puede ver, este aumento de personal es rápidamente absorbido por el incremento de personas usuarias, lo que conlleva un incremento de la carga asistencial por parte de los profesionales.

\subsection{Prestaciones económicas de urgencia social}

En este apartado vamos a analizar la interrelación entre la situación de desempleo (tasa de desempleo

Gráfico 4. Comparación de la evolución de los indicadores de desempleo y número de personas atendidas por profesional

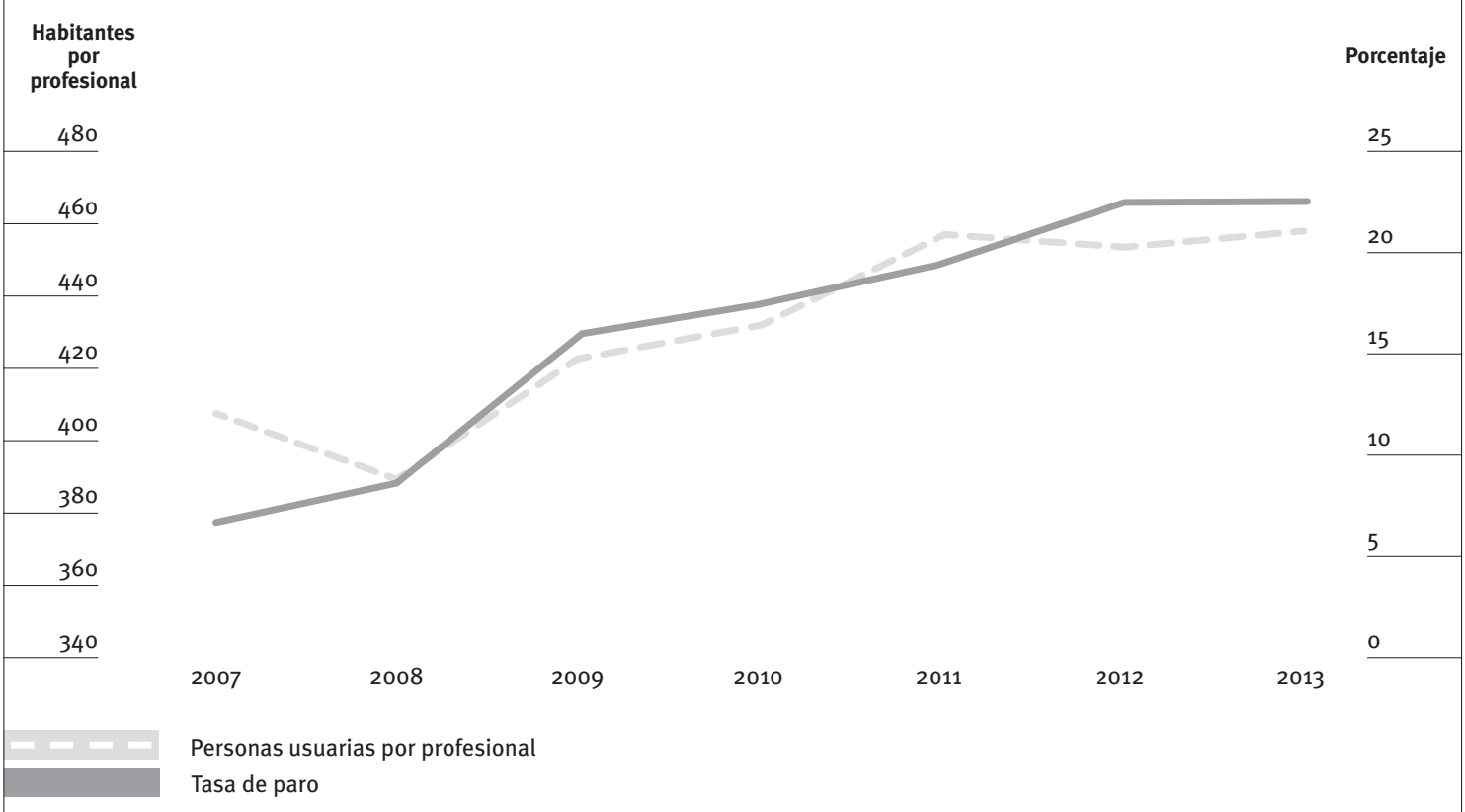

Fuente: Elaboración propia. 
y porcentaje de viviendas familiares con todos los miembros en desempleo), como efecto directo de la crisis económica, con las prestaciones de servicios sociales que tienen componente económico: las ayudas de urgencia social y la renta mínima de inserción (RMI). Dado la serie de resultados de las prestaciones de urgencia social se inicia en 2010, se hará el análisis limitado al periodo 2010-2013. En el Gráfico 5, las líneas continuas muestran los indicadores de desempleo y presentan un trazado prácticamente paralelo ascendente; las líneas discontinuas muestran los indicadores de las prestaciones de servicios sociales y en este caso muestran tendencias divergentes. Mientras que el gasto corriente por familia beneficiaria de las ayudas de urgencia (línea de puntos) sigue la tendencia al alza de los indicadores socioeconómicos, con un incremento del $45 \%$ del gasto por habitante destinado a estas ayudas, la cobertura de la RMI presenta una tendencia a la baja. Parece que la curva de la cobertura de la RMI sea el reflejo en espejo de la curva del porcentaje de hogares con todos los miembros desocupados: a medida que el porcentaje de estos hogares aumenta, el número de RMI disminuye.

\subsection{Servicios sociales de atención domiciliaria}

Los servicios de atención domiciliaria (SSAD) se destinan, fundamentalmente, a la atención de situaciones de dependencia (incluidas o no en la LAPAD) y los resultados no muestran un impacto claro de la situación económica.

Veamos la comparativa entre porcentaje de personas exentas del copago, cobertura de los SSAD en mayores de 65 años y gasto medio de los hogares. En el Gráfico 6 se observa que el fuerte descenso del gasto medio corre paralelo a una mayor aportación en precios públicos (descenso de la tasa de exención), mientras que la cobertura se mantiene prácticamente estable.

\section{Gráfico 5. Relación entre indicadores de desempleo y prestaciones sociales de carácter económico}
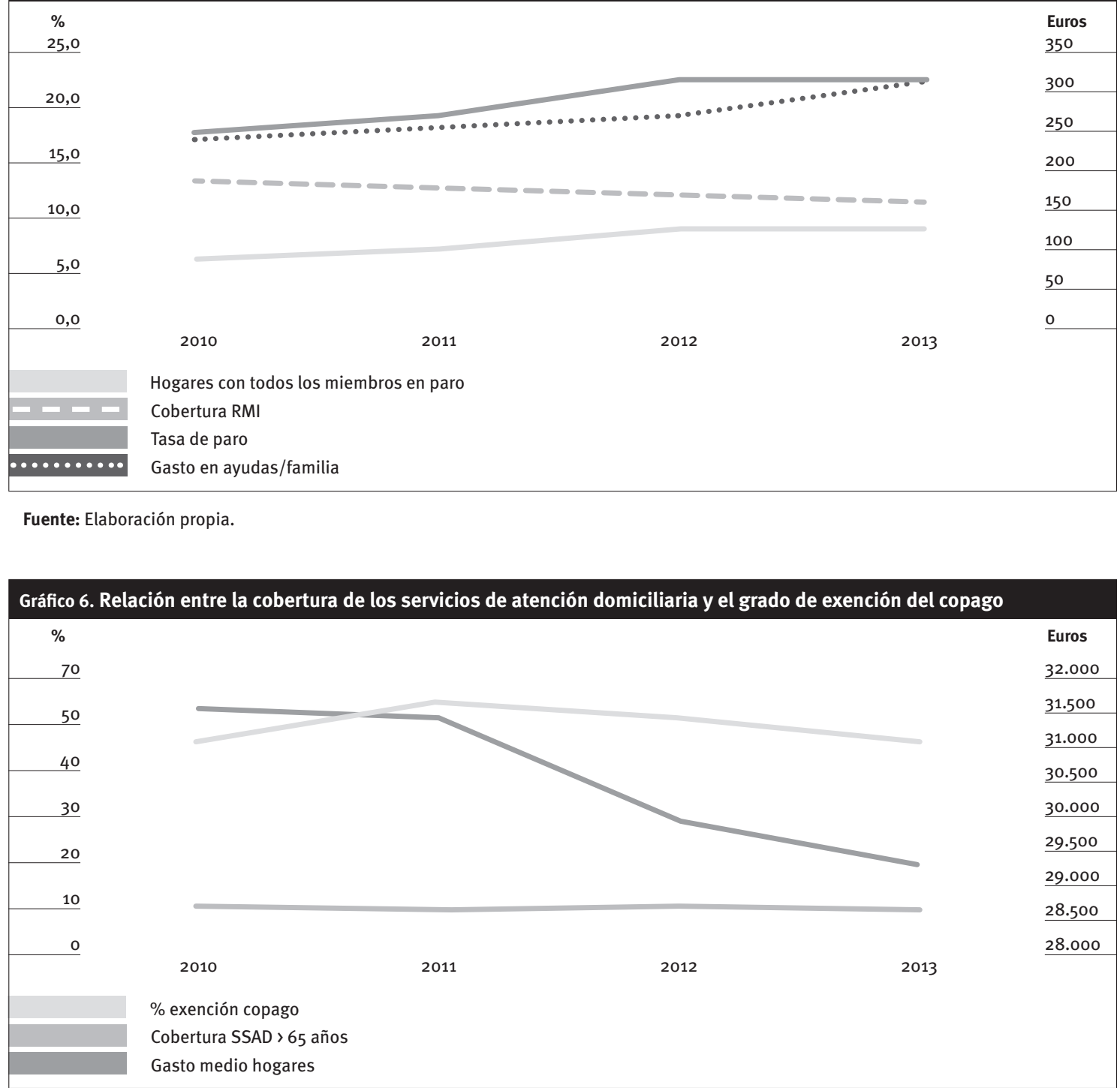
En resumen, los servicios de atención domiciliaria se mantienen estables con una cobertura en torno al $12 \%$, sin que los cambios en la situación económica de las familias parezcan afectarles, pese al incremento de las aportaciones por copago que, recordemos, son limitadas. Es la dotación municipal la que permite esta sostenibilidad, pues la estructura de financiación muestra que son ellos los que sostienen fundamentalmente estos servicios.

\subsection{Centros abiertos}

En primer lugar, queremos analizar la oferta de las plazas en relación con la tasa de riesgo de pobreza en los menores de 16 años $^{10}$ y podemos observar que la curva de la oferta presenta un patrón bastante estable, con pocas modificaciones, mientras que la tasa de riesgo de pobreza sufrió en la primera parte del periodo (2007-2009) un importante aumento y no es hasta los dos últimos ejercicios que las tendencias de las curvas se unifican.

Un segundo análisis muestra que se mantiene la oferta de plazas de centro abierto, pero con un descenso importante de la aportación de los municipios. No hay datos que expliquen esta relación por existencia de otras fuentes de financiación, por ejemplo, o por disminución de los costes, pero, en todo caso, el descenso a la mitad del gasto corriente hace pensar que es difícil una reducción de costes tan importante sin que afecte a la calidad de los servicios prestados.

Gráfico 7. Relación entre la oferta de plazas de centro abierto y la tasa de riesgo de pobreza en la población infantil

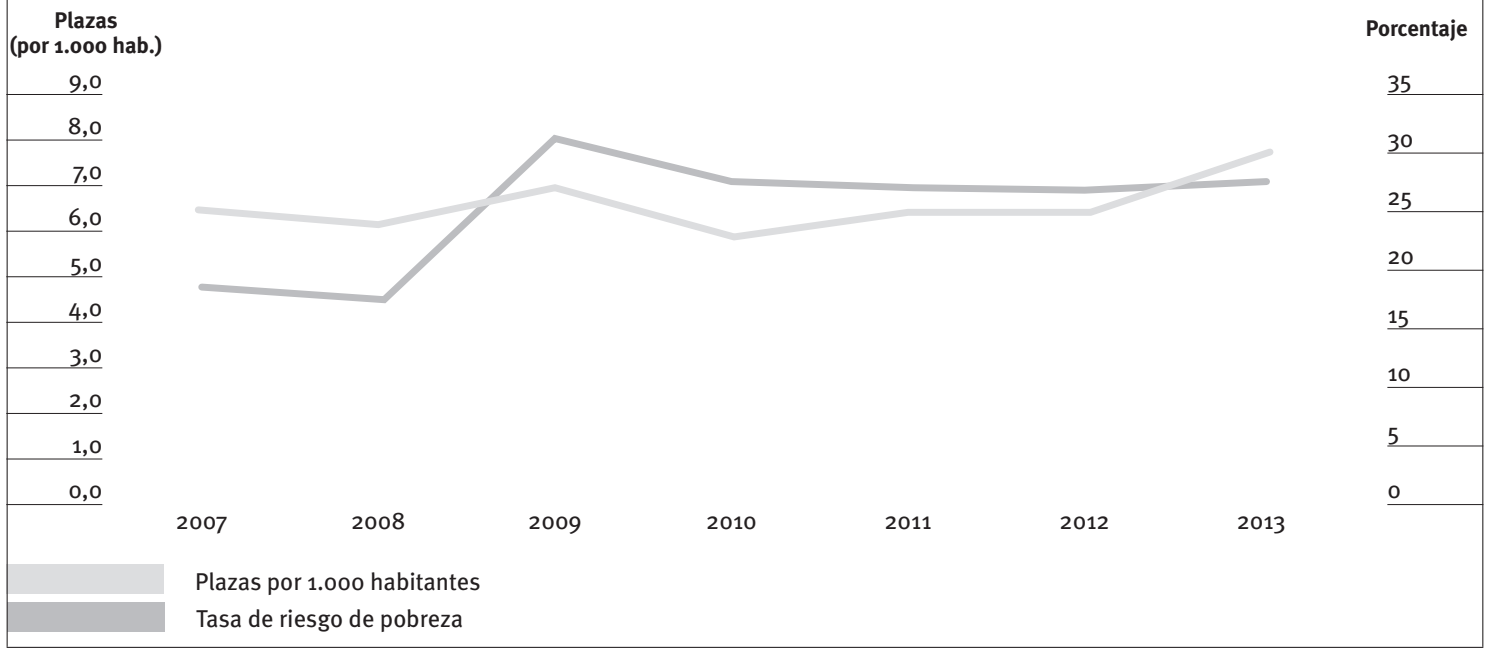

Fuente: Elaboración propia.

Gráfico 8. Relación entre el gasto corriente en euros por habitante en centros abiertos y la ratio de plazas por habitante

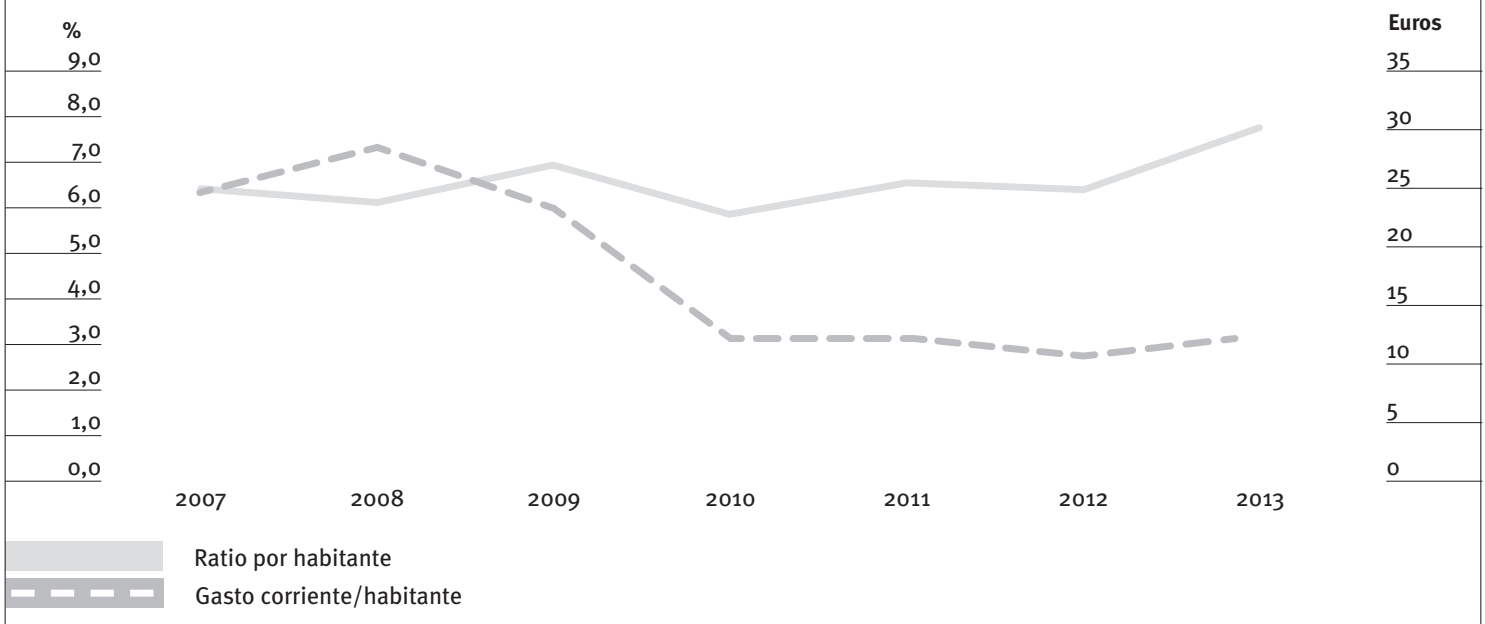

Fuente: Elaboración propia.

${ }^{10}$ La tasa de riesgo de pobreza por edades se clasifica en tres grupos: menores de 16 , de 16 a 64 y mayores de 65 años. En este caso nos centraremos en el grupo de o a 15 años. 


\section{Conclusiones}

El objetivo de este trabajo es identificar y seleccionar los indicadores que pueden medir el impacto de la crisis en los servicios sociales municipales; para ellos, se estudiaron distintos conjuntos de indicadores y se escogió el de los Círculos de Comparación Intermunicipal por la estabilidad de la batería de indicadores y de la metodología, así como por disponer de información a nivel municipal en el periodo elegido, que incluye momentos de crecimiento y de declive económico, de bonanza y crisis.

Las conclusiones analizarán el impacto de los efectos de la crisis en los servicios sociales según esta comparación y acabarán con el análisis de la sensibilidad de los indicadores para medir este impacto.

\subsection{Impacto de los efectos de la crisis en los servicios sociales según los indicadores}

De acuerdo con los indicadores analizados, podemos concluir que los servicios sociales básicos en general, y especialmente los servicios básicos de atención social, han respondido durante la crisis económica (hundimiento del PIB, incremento de la tasa de paro, caída del gasto medio familiar) mediante la disposición de mayores dotaciones de personal que han permitido atender a un número cada vez mayor de personas que requerían atención a sus necesidades sociales. Las representaciones gráficas muestran esa correlación del esfuerzo de los municipios en proveer una mayor dotación a los servicios sociales en términos económicos (gasto por habitante) y en términos de dotación de profesionales (ratio de habitantes por profesional) en una tendencia similar al incremento de la tasa de paro y del porcentaje de hogares con todos los miembros en situación de desempleo. Este incremento en medios económicos y humanos conlleva, lógicamente, el aumento del peso en el conjunto de la organización municipal.

No obstante, cuando nos enfocamos en las distintas prestaciones, los resultados no son tan favorables. Los indicadores de atención domiciliaria no nos permiten inferir a qué orientación de la política social responden, puesto que hay demasiadas variaciones de las que no se puede identificar la causa, si es que hay una causa principal, ya que los indicadores no la muestran.

Por otra parte, una prestación que forma parte de la red asistencial de último recurso, la renta mínima de inserción, muestra un comportamiento contrario al esperado porque disminuye en la medida en que aumenta la necesidad social. En compensación, a partir del momento de inicio de este descenso, se observa la reacción de los municipios mediante el incremento de las prestaciones económicas de urgencia social. Estos resultados nos informan de un modelo de política social que parece alejarse del espíritu de la ley porque se deposita el peso en prestaciones poco reguladas y de concesión variable frente a las prestaciones de derecho subjetivo, como es el caso de la renta mínima de inserción (aunque no se haya modificado este extremo en la norma, se ha suprimido de facto este derecho).

Finalmente, los indicadores sobre centros abiertos muestran una situación preocupante, puesto que se producen incrementos en la cobertura, pero con menores dotaciones económicas y esta situación, que no tiene explicación mediante los indicadores, debería estudiarse para identificar si se está sacrificando la calidad por la cantidad.

\subsection{Sensibilidad de los indicadores para evaluar impactos del contexto económico y social}

Buena parte de los 66 indicadores que forman la batería tiene un fuerte componente estructural y los servicios sociales básicos siguen siendo un servicio fundamentalmente público, de prestación directa desde las Administraciones; por ello difícilmente miden los vaivenes que podrían darse si esos mismos indicadores se hubieran aplicado al sector de la iniciativa social o privada de los servicios sociales. No obstante, pese a esta característica, la interrelación entre los resultados de los Círculos y los indicadores macro y socioeconómicos permite contrastar el papel que tienen asignados los servicios sociales (aquel que las leyes les asignan en cuanto a atender las necesidades básicas y sociales de las personas).

Los indicadores diseñados para los servicios sociales básicos, equipos básicos de atención social y centros abiertos han mostrado la sensibilidad suficiente para mostrar la evolución de la actuación en función del contexto económico tanto en el sentido esperado o establecido en las funciones previstas en las normas, como para identificar aquellos aspectos que presentan incoherencias.

Algunos de los indicadores muestran, al ponerse en relación con el índice de precios al consumo, con la tasa de desempleo o con el gasto medio de las familias, que la acción de los servicios sociales se corresponde con una de las funciones que se le han asignado, porque responden a mayores necesidades con la disposición de más medios y prestaciones. Es el caso de la evolución del gasto por habitante en servicios sociales básicos, que sigue la misma tendencia que la evolución de la tasa de paro, al mismo tiempo que muestra una tendencia contraria a la evolución del PIB; son acciones esperables por parte del sistema.

Sin embargo, otros indicadores han mostrado que la acción que se ha llevado a cabo se aleja de la misión asignada; los datos sobre la renta mínima de inserción (única prestación en la que los municipios solo realizan el papel de gestores, pero en la que no 
tienen capacidad de decisión) muestran resultados de signo contrario al esperado y disminuye su cobertura cuando se producen los incrementos de las situaciones objetivas de necesidad.

La interrelación entre estos datos también permite identificar situaciones que deberían ser objeto de reflexión, por ejemplo, que el mayor incremento del porcentaje de financiación de los servicios sociales mediante el copago (tasas y precios públicos) se produzca coincidiendo con las más altas tasas de paro. Debería ser un indicador que permitiera reflexionar si se produce por un incremento de las personas atendidas o por un incremento de la presión sobre dichas personas, y si esta responde a los objetivos del sistema de servicios sociales.

Los indicadores por sí solos no ofrecen más información que la que las variables muestran, pero debemos recordar que no dejan de ser expresiones numéricas de una variable y que su interpretación forma parte del proceso de evaluación de los servicios. Los Círculos incluyen cuatro indicadores de contexto (población, población extranjera, tasa de paro y renta per cápita) pero a lo largo del informe de difusión pública no se analiza la relación de estos indicadores con los resultados, sino que se limitan a constatar incrementos y decrementos en los resultados en relación con otros ejercicios.

Así pues, los indicadores muestran una información básica que puede y debe ayudar a la toma de decisiones porque, especialmente los indicadores de cobertura y los de esfuerzo económico, han mostrado un grado de sensibilidad bastante notable, pero para ello es imprescindible disponer de análisis de datos cruzados sobre el contexto y la acción de los servicios sociales básicos. Por sí solos, descontextualizados, los resultados no informan de los posibles impactos sobre la sociedad; resultados (outputs) que, por otra parte, deberían disponer de indicadores específicos puesto que prácticamente no existen indicadores que midan outputs (resultados) ni outcomes (impactos). La indefinición de los objetivos de los servicios sociales, incluyendo aspectos que no dependen exclusivamente de su acción (por ejemplo, la cobertura de necesidades básicas) dificulta la evaluación de los impactos. Pero, aún más, los indicadores diseñados parecen centrarse en una sola función de los servicios sociales básicos, atender las necesidades básicas (de subsistencia) y desde una perspectiva muy economicista, mientras que la atención a las necesidades relacionadas con la autonomía personal y las relaciones interpersonales y sociales (incluidas en el objetivo asignado a los servicios sociales) no disponen de indicadores para su medida.

En conclusión, los indicadores muestran sensibilidad para medir limitadamente la acción de los servicios sociales básicos. El análisis conjunto de indicadores muestra una realidad contradictoria: no siempre las políticas sociales aumentan cuando aumenta su necesidad. 
DELOITTE (2008): El Servicio de Atención a Domicilio (SAD), Barcelona.

DEPARTAMENT D'ACCIÓ SOCIAL I CIUTADANIA-GENERALITAT DE CATALUNYA (2010): Decret 142/2010, d'11 d'octubre, pel qual s'aprova la Cartera de Serveis Socials 2010-2011, Barcelona, Govern de la Generalitat de Catalunya.

DIPUTACIÓ DE BARCELONA (2014): Guia dels indicadors dels cercles de comparació intermunicipal de Serveis Socials, [khttp://www.diba.cat/ documents/446869/99305577/Serveis-Socials. pdf>].

FUSTIER, N. (2006): Círculos de comparación en servicios sociales, Barcelona, no publicado.

- (2008): El benchmarking en la millora de la qualitat dels Serveis d'Atenció Domiciliària, Barcelona, inédito.

- (2015): “¿Pueden los indicadores existentes medir el impacto de la crisis económica en los servicios sociales básicos? Un análisis de los indicadores en la provincia de Barcelona (2007 - 2013)", [khttps://www.researchgate. net/publication/301351716_Pueden_ los_indicadores_existentes_medir_el impacto_de_la_crisis_economica_en_los_ servicios_sociales_basicos_Un_analisis_ de_los_indicadores_en_la_provincia_de Barcelona_207_-_2013〉].

GÓMEZ I SERRA, M. (2000): Els serveis socials i la seva avaluació, Barcelona, Universitat de Barcelona.

IDESCAT (2014): Encuesta de población Activa. Metodología, [«http://www.idescat.cat/treball/epa?tc=8\&id $=\mid C 13 \&$ lang $=e s \& a c=4\rangle]$, consultado el 29 enero de 2017.
- (s/f): Producte interior brut (PIB). Base 2008, Metodologia [<http://www.idescat.cat/economia/ inec?tc $=7 \&$ id $=0007\rangle]$, consultado el $21 \mathrm{de}$ enero de 2017.

INE (2007): Encuesta de Presupuestos Familiares. Metodología. S.l. [<http://www.ine.es/ metodologia/t25/t2530p458.pdf〉], consultado el 29 de enero de 2017.

KRUGMAN, P. (2013): “¿Una depresión permanente?”, El País, Madrid; 24 de noviembre de 2013 [rhttp://economia.elpais. com/economia/2013/11/22/ actualidad/1385142585_896442.html s].

LLEI 12/2007, D'11 D'OCTUBRE, DE SERVEIS SOCIALS (2007): Barcelona, Parlament de Catalunya.

LLEI ORGÀNICA 6/2006, DE 19 DE JULIO, DE REFORMA DE L'ESTATUT D'AUTONOMIA DE CATALUNYA (2006), Barcelona.

MATULIC, V. y FUSTIER, N. (2008): "Los servicios sociales básicos en la nueva ley de servicios sociales de Cataluña", Servicios Sociales y Política Social, no. 83 , pp. $127-135$.

ROSENDE, F. y BENGOLEA, J.M. (1984): “Teoría de los ciclos y la crisis económica actual", Cuadernos de economía, n. 63, vol. 21, pp. 207-226 [shttps://repositorio.uc.cl/ bitstream/handle/11534/16395/000322810. pdf? sequence $=1$ \&isAllowed $=y>$ $>$.

UXÓ GONZÁLEZ, J. (sin fecha): “Crisis económica”, Expansión, Madrid [<http://www.expansion. com/diccionario-economico/crisis-economica. html>].

VV. AA. (2013): 12a edició del Cercle de comparació intermunicipal de serveis socials; Barcelona. 